\title{
The streamwise drag-reduction response of a boundary layer subjected to a sudden imposition of transverse oscillatory wall motion
}

\author{
Sylvain Lardeau ${ }^{1}$ and Michael A. Leschziner ${ }^{2}$ \\ 1) CD-adapco, 200 Shepherd's Bush Road, London W6 7NL, \\ United Kingdom \\ ${ }^{2)}$ Department of Aeronautics, Imperial College London, South Kensington Campus \\ London SW7 2AZ, United Kingdom
}

(Dated: 6 June 2013)

\begin{abstract}
A DNS study is presented, which examines the response of a spatially developing boundary layer to oscillatory spanwise wall motion imposed over a limited streamwise stretch. At the heart of the study is the dependence of the streamwise variations in skin friction and turbulence properties on the period of the oscillatory motion, with particular emphasis placed on the behaviour downstream of the start of the actuation. The friction Reynolds number just upstream of the actuation is $R e_{\tau}=520$, and the wall-scaled actuation period, $T^{+}=T u_{\tau}{ }^{2} / \nu$, covers the range 80-200. In contrast to channel flow, the present configuration allows the processes during the transition stretch from the unactuated state to the low-drag state and the recovery from the low-drag state to be studied. Attention focuses primarily on the former. Results are included for the time-averaged turbulent stresses, their budgets and PDFs, as well as a range of phase-averaged properties. The study brings out, for low-drag conditions, a number of features and processes that are common with those in actuated channel flow, but suggests that the maximum drag-reduction margins are lower than those in equivalent channel flow, and that the optimum actuation period is significantly shorter. The transition to the low-drag state occurs over about 5 boundary-layer thicknesses, and is characterized by substantial oscillations in all phase-averaged properties. These oscillations, provoked at the start of the spanwise motion, propagate convectively as waves and decay as the low-drag state is approached. The interactions contributing to the oscillations are discussed as part of the analysis of phase-averaged quantities.
\end{abstract}

Keywords: Turbulent drag reduction; Skin friction; Boundary layer; Spanwise wall oscillations, Direct Numerical Simulation

\section{INTRODUCTION}

It is well established, computationally as well as experimentally, that the imposition of oscillatory spanwise wall motion, either in streamwise-homogeneous or streamwise-wavy mode, causes substantial reductions in turbulent skin-friction drag, if the actuation is effected within judiciously chosen bands of parameters. Almost all knowledge on this subject has been derived from DNS studies of channel flow and a few experimental studies on boundary layers, both at relatively low values of the Reynolds number - mostly in the range $R e_{\tau}=200-400$. A representative example is the DNS study of Quadrio et al. ${ }^{1}$, in which it is demonstrated that gross drag reductions of up to $47 \%$ can be achieved in channel flow at a friction Reynolds number, $R e_{\tau}=200$ at near-optimum actuation frequency and wave length. In the case of streamwise-homogeneous wall motion, the maximum drag-reduction margin is somewhat more modest, around $37 \%$ at $R e_{\tau}=200$ and wall-scaled actuation period $T^{+}=100$, dropping to around $30 \%$ at $R e_{\tau}=1000$ at a rate roughly proportional to $R e_{\tau}^{-0.2}$ (Touber and Leschziner ${ }^{2}$ ). In all cases, the range of effective actuation parameters is narrow, however. Indeed, the drag can increase above that of the unactuated case in unfavourable conditions. Thus, Quadrio et al. ${ }^{1}$ obtained, again for $R e_{\tau}=200$, a drag increase of around $20 \%$ above the unactuated level within a band of oscillation frequency and wave length lying fairly close to the most effective range. 
Experimental investigations on boundary layers by Laadhari et al. ${ }^{3}$, Choi et al. ${ }^{4}$, Choi ${ }^{5}$, Di Cicca et al. ${ }^{6}$ and Ricco ${ }^{7}$ all show a qualitatively similar response to oscillatory wall motion, downstream of a stretch of around 3-5 boundary-layer thicknesses within which the drag reduces towards its equilibrium level. However, substantial differences in the maximum drag-reduction margins are reported - ranging from $23 \%$, measured by Ricco ${ }^{7}$, up to $45 \%$, obtained by Choi and Graham ${ }^{8}$ - and this variability reflects (if experimental errors are presumed modest) significant differences in the wall-velocity amplitude, the Reynolds numbers and also the actuation periods among the studies. Boundary-layer simulations by Skote ${ }^{9,10}$ at $R e_{\tau}$ no higher than 270 , at actuation periods close to the value $T^{+}=$ 100 , generally regarded as close to optimum in low-Reynolds-number channel-flow, yielded maximum drag-reduction margins of 18\%-37\%, confirming a strong dependence on the wallvelocity amplitude and, to a lesser extent, on the Reynolds number, the latter albeit within the narrow range $R e_{\tau}=200-270$.

Regardless of the precise drag-reduction margins, the above observations are of significant interest in the context of efforts to reduce fuel consumption in vehicular transport, especially civil aviation - although the engineering challenges of realizing oscillatory spanwise motion in practical configurations are clearly formidable. Equally important, the potential net benefits are obviously much lower than the gross levels mentioned above, due to the power input needed for the actuation - a subject discussed in relation to several flow-control techniques by Kasagi et al. ${ }^{11}$ and Frohnapfel et al. ${ }^{12}$. For the specific case of oscillatory wall motion imposed as streamwise waves, Quadrio et al. ${ }^{1}$ show that the maximum gross reduction of $45-47 \%$, at $R e_{\tau}=200$ and in the actuation conditions mentioned above, reduces to a net benefit of $15-17 \%$ if the actuation power is taken into account. Yet, even reductions in the range of a few percentage points provide powerful motivation for pursuing studies on wall-oscillation-driven drag decrease, with a view to related technologies being realized in the long term. In the case of streamwise-homogeneous actuation, the net effect has been shown by Quadrio and Ricco ${ }^{13}$, Quadrio et al. ${ }^{1}$ and Frohnapfel et al. ${ }^{12}$ to be negative for most actuation conditions investigated so far, except for low values of the wall-velocity magnitude at which the gross drag-reduction margins are low. However, this actuation mode is nevertheless of considerable interest, as it provides an attractively simple background against which the influential fundamental interactions that lead to substantial gross drag reductions can be illuminated.

Many of the past studies, especially those based on DNS, focus primarily on the quantification of the drag-reduction level and its dependence on the actuation parameters, while only a few seriously probe the underlying turbulence mechanisms. A review of the pertinent literature up to 2012 is included in a recent paper by Touber and Leschziner ${ }^{2}$, and another summarizing work prior to 2003 is given in Karniadakis and Choi ${ }^{14}$. Studies investigating fundamental interactions from a variety of viewpoints - among them Di Cicca et al. ${ }^{6}$, Laadhari et al. ${ }^{3}$, Choi et al. ${ }^{15}$, Ricco ${ }^{7}$ and Touber and Leschziner ${ }^{2}$ - variously show that the Stokes strain induced by the actuation disrupts the streaky near-wall structure, causes a periodic re-orientation of the streaks - with significant weakening between reorientation phases, and reduces the intensity of quasi-streamwise vortices, or ejections and (to a lesser extent) sweeps, thus ultimately damping the turbulent mixing in the viscous sublayer. The recent DNS study of Touber and Leschziner ${ }^{2}$, in particular, presents a wealth of statistical and structural data, mainly at $R e_{\tau}=500$, that allowed deeper insight to be gained into some key fundamental mechanisms, but only in near-equilibrium conditions at which the actuation had become fully effective in reducing the drag to its final low value. Minor phase-dependent variations in the drag and turbulence fields around the low-drag state, especially in non-optimum oscillation frequencies, were exploited by Touber and Leschziner ${ }^{2}$ to identify that the spanwise distortions of the streaks created regions across the viscous sublayer in which the wall-normal and shear stresses experiences severe damping in particular phases of the actuation cycle, while other regions experience more minor amplification. This response was linked to the observed dependence of the streak attenuation, amplification and reorientation on the rate of change of the total strain in specific portions of the Stokes layer. In parallel, associated linear analyses by Blesbois et al. ${ }^{16}$, employing the 
Generalized Optimum Perturbation technique, revealed the optimum actuation period to be linked to the streak-amplification time scale. Thus, following streak damping by rapid variations in the near-wall strain angle, regeneration is inhibited by the period over which the strain lingers (i.e. changes little in its orientation) being too short relative to the streakamplification time scale. Finally, a recent analysis of phase-averaged data at $R e_{\tau}=1000$ and two actuation frequencies, one being close to optimum value, provides a picture that is broadly consistent with that derived by Touber and Leschziner ${ }^{2}$ for $\operatorname{Re}_{\tau}=500$.

The imposition of streamwise periodicity in channel-flow DNS clearly precludes the spatial progression of the drag-reduction process, following the onset and termination of the actuation, to be examined and understood. There is no reason to suppose that the processes in the low-drag state apply to these transitional portions. In fact, two previous studies ${ }^{17,18}$, which examined the temporal evolution of the drag-reduction process in channel flow following the sudden imposition of spanwise oscillations over the entire length of a fully-established and streamwise-homogeneous turbulent base flow, reveal a behaviour that is not observed within the low-drag state, and suggest that the imposition of the spanwise oscillation at a fixed streamwise location of a boundary layer might also provoke correspondingly peculiar features. For example, the drag and turbulence intensity were observed to reduce in a nonmonotonic fashion, with turbulence production experiencing overshoots during the transient path towards the low-drag state, the final level being attained within about 3 oscillation periods. The study by $\mathrm{Xu}$ and Huang ${ }^{18}$ also suggests that this non-monotonic behaviour is associated with an anomalous response of the pressure-strain interaction in the shear-stress balance. However, the details of the mechanism are unclear. Moreover, transient-response studies pose two potentially important uncertainties, especially if used in lieu of the transitional region of an actuated boundary layer. First, fluctuations derived in the transient region are not purely stochastic and cannot be separated, in a phase-averaged sense, from the non-turbulent transient motions. Second, the rapid formation of the Stokes layer, from a zero thickness to its equilibrium low-drag state during the transient process, cannot generate the expected non-homogeneous features in the streamwise direction, and this may provoke a response that is not representative of the transition stretch in a boundary layer.

To the authors' knowledge the only computational investigations of the response of a spatially-evolving boundary layer, subjected to oscillatory wall motion over a limited streamwise stretch of the boundary layer, are those of Skote ${ }^{9,10}$, already mentioned above. These studies, at $R e_{\tau}=200$ and 270 and $T^{+}=118$ or 132 (depending on the wall-velocity amplitude), provide information on the time-averaged properties along the boundary layer, the latter study also analyzing the variation of some properties in time - derived as equivalent to the streamwise distance divided by a convection velocity at around $y^{+}=10$. It is important to stress the fact that this equivalence, meant to link the boundary layer to transients in actuated channel flow, does not resolve any phase-dependence, for this requires phase-averaging to be undertaken to separate the stochastic turbulence from the periodic component of the motion. This limits the insight that the study can provide in respect of the transition process. It is also the reason why the study cannot reveal the type of non-monotonic behaviour observed in the transient response of channel-flow to the sudden onset of wall oscillations.

In contrast to Skote's work, the present study includes a focus on the response of the phase-averaged turbulence properties to the oscillatory motion along the transition region in a boundary layer at $R e_{\tau}=520$, relative to Skote's highest value of 270 . The study is closely linked, in terms of both the actuation parameters and the analysis performed, to the channel-flow work of Touber and Leschziner ${ }^{2}$ at $R e_{\tau}=500$. Apart from the fundamental aspects that the present configuration offers, the study is motivated by the fact that this is the most likely scenario to feature in any practical realisation of drag reduction by wall oscillations. Of the two streamwise stretches that follow the onset and termination of the actuation, the former is of greater interest and also greater complexity. For this reason, the present paper focuses primarily on this region. 


\section{COMPUTATIONAL PROCEDURE}

The flow under consideration is a flat-plate, zero-pressure-gradient boundary layer evolving over 45.8 inflow boundary-layer thicknesses $\delta_{0}$. Spanwise wall oscillations were imposed between positions $x_{0}=6.64 \delta_{0}$ and $x_{1}=30.2 \delta_{0}$, with a hyperbolic-tangent ramp-up over $0.2 \delta_{0}$ upstream, to study the processes during the steep drop and rise of the drag (although only the former is analyzed in detail herein). The spanwise actuation is thus described by:

$$
W_{\text {wall }}(x, t)=W_{\max }^{+} u_{\tau} R_{\text {up }}(x) R_{\text {down }}(x) \sin \left(2 \pi \frac{t-T_{0}}{T^{+}} \frac{u_{\tau}^{2}}{\nu}\right)
$$

where $T_{0}$ is the time at which the actuation is initiated and the functions providing the ramping up and down are given, respectively, by

$$
R_{\text {up }}(x)=\frac{1}{2}\left[1+\tanh \left(10 \frac{x-x_{0}}{\delta_{0}}\right)\right], \quad R_{\text {down }}(x)=\frac{1}{2}\left[1-\tanh \left(10 \frac{x-x_{1}}{\delta_{0}}\right)\right]
$$

The ramping-up process is only designed to avoid numerical oscillations, and its precise character is of negligible consequence to the ensuing flow-physical processes, as confirmed by preliminary simulations. Within the short streamwise ramp-up segment, the only actuation parameter that varies is the amplitude of the oscillation, and this is rising quickly. The influence of the amplitude on the drag-reduction behaviour is considered in several past studies, albeit only in respect of the final low-drag level. For example, Skote ${ }^{10}$ and Choi et $a l^{15}$ show that halving the amplitude from the present level of $W_{\max }^{+}=12$ still yields $60-70 \%$ of the drag-reduction at the higher amplitude. This suggests, on its own, that the impact of the reduced amplitude during the ramp-up process is likely to be low, except in the very beginning, during an extremely short streamwise portion of the ramp-up segment. A further argument leans on the observation (see Fig. 2) that the initial decline in drag does not depend on the actuation period - i.e. on the streamwise rate of change in the spanwise velocity. During this phase, the Stokes layer is very thin, and the very high strain in this layer disrupts the turbulence regeneration near the wall, irrespective of the time scale over which the spanwise velocity increases. Varying the rate of rise in the oscillation amplitude with streamwise distance in the ramp-up region also implies variations in the streamwise rate of change in the spanwise velocity. However, as argued, the rate of change is evidently not of significant influence on the initial drag decline, so the expectation is that the details of the ramp-up process will be insignificant too, especially if the ramp-up distance is short, as it is here.

DNS-level computations were performed on a $2048 \times 160 \times 192$ mesh, with near-wall cell dimensions $\Delta x^{+}, \Delta y_{\min }^{+}, \Delta z^{+}=8,1,8$. This level of resolution is comparable to that of most published channel-flow DNS studies investigating the effects of spanwise wall motion on the $\mathrm{drag}^{2}$, except that the present spanwise resolution is better than that in most earlier studies. The adequacy of this resolution is discussed in considerable detail, in terms of several quality indicators, in Lardeau and Leschziner ${ }^{20}$ and Bentaleb et al. ${ }^{21}$ who report simulations with the same numerical scheme for boundary layers with properties very similar to that considered herein, but focusing on entirely different physical issues. The present simulations were run on 960 processors on the UK national facility HeCTOR..

Fully turbulent inflow conditions at the displacement-thickness Reynolds number $R e_{\delta^{*}}=$ 1230, where $\delta^{*}$ and $R e_{\tau}=523$ were prescribed by sampling from a precursor simulation performed over a period of 1450 time units, $\delta_{0} / U_{0}$, evaluated at the inlet. Over the length of the computational domain, the free-stream velocity varies by less than $0.5 \%$ of the reference (inflow) free-stream velocity $U_{0}$. Furthermore, the predicted streamwise variation of the momentum thickness with $R e_{\tau}$ for the baseline case was found to agree closely with data by Jiménez et al. ${ }^{22}$, Schlatter et al. ${ }^{19}$ and $\mathrm{Wu}$ and Moin ${ }^{23}$. Second moments and the turbulence-energy budget derived from the database are shown in Fig. 1, in comparison with data for a similar boundary layer reported by Schlatter et al. ${ }^{19}$.

The simulations were run, without wall oscillations, for a period of $t=200$ time units, $\delta_{0} / U_{0}$, during which the initial conditions were evacuated. Statistical sampling of the un- 
actuated boundary layer was then performed over the next 300 time units. Spanwise wall oscillations at the periods $T^{+}=T u_{\tau}^{2} / \nu=80,100,120$ and 200, with $u_{\tau}$ taken just upstream of the start of the actuation, were then started at 500 time units, and statistical sampling of mean and phase-averaged data commenced at 575 time units, over a subsequent period of 620 time units, corresponding to 120 actuation cycles in the case of $T^{+}=100$. For $T^{+}=200$, only 45 actuation cycles have been used for averaging.

The choice of the oscillation periods was guided by results for channel-flow simulations, which suggest that the value $T^{+}=100$ yields a close to maximum drag-reduction margin at $R e_{\tau}=200-1000^{2}$. It is emphasized, however, that it was not the primary objective of the present study to find the optimum actuation parameters that would result in the maximum drag reduction for the present configuration. Although Skote ${ }^{10}$ suggests that the drag-reduction margin in a boundary layer, at $R e_{\tau}=200$, is only slightly lower than in channel flow at, essentially, the same oscillation period (around $T^{+}=100-130$ ), there is no compelling reason to assume that the optimum actuation periods are identical in both configurations, or that the maximum drag-reduction margins are close to each other as the Reynolds number increases. One of several possible sources of differences between the two types of flow is the different turbulence structure in the outer part of the boundary layer, beyond $y^{+} \approx 100$; this issue will be revisited later.

Phase-averaged data were only recorded for $T^{+}=100$ and 200, with each oscillation period being divided into 36 equal-sized bins. While these data are reasonably well converged, distributions presented below will be seen to feature modest undulations. However, it must be borne in mind that, in marked contrast to channel flow, no streamwise averaging is possible in a boundary layer, nor can mid-channel mean-flow symmetry be exploited here. Hence, the sample size contributing to phase-average data is quite limited, and complete convergence would require integration over many hundreds of actuation cycles at untenable computing costs.

\section{RESULTS}

\section{A. Mean-flow properties}

Attention focuses first on time-averaged characteristics given in Figs. 2-8. Where results are normalised by the friction velocity, its value is taken just upstream of the onset of actuation. While scaling by the local friction velocity might be regarded as more appropriate, on physical grounds, the present scaling choice is deliberate and rooted in the wish to highlight the actual response, essentially in terms of magnitude, of the flow properties to the actuation. This is in contrast to efforts designed to bring out near-wall universality, which does not prevail, in any event, when actuation is present (see Touber and Leschziner ${ }^{2}$ ).

Fig. 2 gives the streamwise variations of the skin friction at different actuation periods, relative to the unactuated level, the upper plot providing a complete view over the entire boundary layer and the lower restricted to the near-field transition region following the onset of actuation. To identify the relationship between $C_{f}$ and the turbulence field, Fig. 3 shows variations of the streamwise normal stress and the shear stress in the transition region along the line $y^{+}=10$. The particular choice of this wall distance is guided by channel-flow studies $^{2}$, which suggest that the effects of the actuation on the turbulence properties in the upper portion of the viscous sublayer are closely linked to the drag-reduction process. In particular, the stochastic streamwise normal stress and its production rate reach their respective maximum values at around $y^{+}=10-15$, consistent with the observation that the near-wall streaks are most pronounced at this wall distance. Because of the use of $u_{\tau}$ at the location ' 0 ' for scaling $y$, the actual value of $y^{+}$is somewhat lower than 10 , especially at low values of $T^{+}$. However, this does not affect significantly the largely qualitative conclusions derived from the variations. To gain a view of the effects of the actuation on the turbulent stresses across the boundary layer, Fig. 4 shows, for $T^{+}=100$ and 200 only, profiles of the streamwise normal stress and the shear stress at the streamwise locations ' 1 ', ' 3 ' and ' 6 ', at 
the first two of which $C_{f}$ and the stresses drop especially steeply following the onset of the actuation.

The results convey the rapid reduction in drag, and turbulence, after the onset of the actuation, with $75 \%$ of the drag-reduction margin occurring within around $3 \delta_{0}$, and the maximum margin of $25 \%$ reached at $T^{+}=80$ at around $5 \delta_{0}$ after the start of the actuation.

Although not being the focus of this paper, it is interesting to compare this value with previous data. First, Ricco ${ }^{7}$ report an experimentally derived drag reduction of approximately $23 \%$ at $T^{+}=67$ and Reynolds number and wall-velocity amplitude very similar to those adopted herein. In contrast, Skote ${ }^{10}$ obtained a reduction of almost $30 \%$, but at a much lower Reynolds number, $R e_{\tau}=200$. It thus appears that the present level of $25 \%$ is entirely realistic, but could increase further, marginally, if $T^{+}$is reduced to 60-70. In any event, the present results imply that the drag-reduction levels in boundary layers are somewhat lower than those achieved in channel flow, at corresponding actuation parameters, at least at elevated Reynolds numbers. Also, the maximum levels are attained at higher actuation frequencies, relative to $T^{+}=100-120$ in channel flow. The latter difference is accentuated if the streamwise decline of $u_{\tau}$ in the unactuated boundary layer is taken into account, for this decline tends to depress $T^{+}$. The origin of these differences is not clear at this stage. However, one possible source lies in significant differences in the turbulence structure of the outer region of the respective boundary layers beyond $y^{+} \approx 100$. One aspect of these differences is that the length scales used in $R e_{\tau}$ - the half-channel height and the boundary-thickness, respectively - may not be physically equivalent. A more appropriate length scale might be the thickness corresponding to the edge of the log-law region. However, this is not likely to be a decisive issue, as the sensitivity of the drag-reduction margin to the Reynolds number is modest. A more intriguing aspect is the lower frequency of the optimum actuation. This points, prima facie, to flow-related differences in the streakdecay and -regeneration time scales during the actuation cycle. It is known (see Touber and Leschziner ${ }^{2}$ ) that the response of the near-wall streaks to the actuation is affected by foot-printing by outer large-scale motions, but whether this interaction plays a role in relation to the differences in the optimal actuation period is not clear.

If it is accepted that the primary effects of the Stokes strain on the turbulence properties associated with the drag behaviour occur in the upper portions of the viscosity-affected sublayer, around $y^{+}=8-12$, it follows, subject to $U^{+}=y^{+}$, that the time scale corresponding to $5 \delta_{0}$ thicknesses is approximately $2-3 T^{+}$. This values agrees well with observations of how fast $C_{f}$ approaches the low-drag state when a channel flow is subjected to a sudden imposition of spanwise oscillatory motion ${ }^{17,18}$ - although caution is required in leaning upon this analogy for reasons discussed in the introduction.

Alongside the reduction in $C_{f}$, there is a strong decline in turbulence activity in the upper portion of the viscous near-wall layer (Fig. 3). Thus, both the streamwise-normal and shear stresses - the former representative of streak intensity - drop precipitously by up to $30 \%$ within around $0.3 \delta_{0}$, and by more than $50 \%$ after $3 \delta_{0}$ at $T^{+}=80$. This disproportionately large reduction reflects a combination of a general decline in turbulence activity across the entire near-wall layer and a thickening of the viscous sublayer (in terms the true local $y^{+}$). This results in the true wall-scaled distance corresponding to the nominal level $y^{+}=10$ shifting to lower parts of the viscous sublayer as $T^{+}$is reduced. A remarkable trend at $T^{+}=200$, seen in both the skin-friction and the stresses, is a recovery towards the baseline level following an initial drop in skin friction and turbulence. This behaviour reflects adverse effects arising from the Stokes strain penetrating into and beyond the buffer region above the viscous sublayer and, at the same time, the declining strain within the critically important layer around $y^{+}=10$. This penetration will be highlighted later when phase-averaged results are discussed. With the optimum $T^{+}$value being around 70 in the boundary layer, $T^{+}=200$ may be assumed equivalent, in terms of drag-reduction effectiveness, to a channelflow value of $T^{+} \approx 300-400$, and this value has been observed to produce a very small drag decrease, at least at $R e_{\tau}=200$ (see Fig. 1 in Touber and Leschziner ${ }^{2}$ ), whilst the shearstress production rate exceeded that in the unactuated flow. Aspects of this interaction will be discussed below by reference to stress-budget considerations. 
Profiles of the streamwise-normal and shear stresses at the positions ' 1 ', ' 3 ' and ' 6 ', for $T^{+}=100$ and 200 are given in Fig. 4 and show that both stress components initially decline at the same rate for both actuation periods, with the decline being most pronounced within $y^{+}=10-30-$ higher values within this range pertaining to the shear stress - while the outer layer, beyond $y^{+}=100$, is hardly affected in the initial portion of the transition region. The behaviour is quite different at location ' 6 ', and parallels that shown earlier in Fig. 3 for the stress components at $y^{+}=10$. In particular, at $T^{+}=200$, both stresses recover, especially across the viscous sublayer, with $\overline{u v}$ virtually returning to its baseline level below $y^{+}=10$. Again, this behaviour is associated with the penetration of the Stokes layer into the outer region above the viscous sublayer. The effect of the actuation persists beyond the viscous sublayer, and this is consistent with the finite, though small, drag reduction margin along the entire stretch within which the boundary layer is actuated (Fig. 2). Budgets for the streamwise-normal and shear stress components across the boundary layer are expected to give some useful indications on the processes driving the rise in shear stress, in particular, and these are considered below.

One expectation is that the primary process that would reflect the variations of the stresses is the production. Whether this expectation accords with reality is conveyed in Fig. 5. The variations show some anticipated features, but also some unusual, if not contradictory, behaviour. As expected, the production of $\overline{u^{2}}$ declines progressively as $T^{+}$ decreases. The decline relative to the baseline level is broadly commensurate with the decline of the stress itself, except for the case $T^{+}=200$, which is far above the optimum period. The mean production of $\overline{u u}$ is $P_{u u}=-\overline{u v} \partial U / \partial y$. While the velocity gradient at $y^{+}=10$ is observed (but not shown here) to rise immediately following the actuation, dropping slowly thereafter, this variation is not large - not exceeding $10 \%$ of the level upstream of the actuation, as will be shown later - and this is the reason why $P_{u u}$ follows fairly closely the trends of the shear stress. Surprisingly, this is not the case with the shear-stress production $P_{u v}$. In particular, for $T^{+}=200$, the production actually increases beyond the baseline level, in contradiction to the behaviour of the shear stress itself. As this production is $P_{u v}=-\overline{v^{2}} \partial U / \partial y$, there are two possible physical interactions that may explain this behaviour. First, as $T^{+}$increases, the increasing cyclic disturbances, due to the unsteady strain penetrating into the buffer layer and the turbulent layer above, causes increasingly influential periodic (non-stochastic) $\tilde{v}$-fluctuations that lead to a significant elevation of the total (stochastic + periodic) of $\overline{v v}$. Results included later will turn out to negate this proposition. Alternatively, the stochastic wall-normal-stress component may be elevated as a result of the Stokes layer penetrating into the region above the viscosity-affected layer. Indeed, other simulations show that the imposition of a uni-directional spanwise wall motion on an initially two-dimensional boundary layer leads initially to a decline in the wall-normal stress, but eventually to a rise above the baseline state as a consequence of the spanwise strain penetrating into the buffer region and beyond. Increases of the stochastic wall-normal stress and the shear-stress production above the respective baseline levels have also been observed by Touber and Leschziner ${ }^{2}$ at $R e_{\tau}=200$ and $T^{+}=400$, despite a positive (though modest) drag-reduction margin. Second, the production must be counteracted by other budget contributions that have to rise in harmony with the production. In a canonical boundary layer, there is virtually a balance between shear-stress production and the pressure-velocity interaction $\phi_{u v}=-\overline{u \partial p / \partial y}-\overline{v \partial p / \partial x}$. This may not necessarily be the case in the actuated boundary layers. However, as shown in Fig. 5, $\phi_{u v}$ follows $P_{u v}$ closely, except for a steep spike immediately after the onset of actuation, and the former continues to be the principal term balancing the production. In fact, it more than balances the production, thus tending to drive down the magnitude of the shear stress, the difference being made up mainly by convection. Thus, despite the production tending to drive the shear-stress magnitude above the baseline case, other terms in the balance lead to the shear stress remaining below the baseline level.

Partial budgets for the shear stress at locations '1', '3' and '6', highlighting the most important interactions, are shown in Fig. 6. As noted earlier, the budget is dictated largely by a balance between production and pressure-velocity interaction. At location ' 1 ', the 
production drops, but the pressure-velocity term rises well above the baseline level at both actuation periods. As suggested by the steep peak in Fig. 5, this rise is confined to a very short region immediately downstream of the onset of the actuation; indeed, the budgets at location ' 3 ' no longer features this excess, and the production as well as pressure-velocity interaction are below the respective baseline levels, at both actuation periods. The difference between the high pressure-velocity term and the lower production term is made up mostly by convection, associated with the initial rapid streamwise depression in the shear stress. At location '6', the unusual behaviour at $T^{+}=200$ again comes to the fore. Thus, both production and the pressure-velocity interaction at $T^{+}=200$ exceed the respective baseline levels, within $y^{+}=50$, and this is consistent with the behaviour already highlighted earlier by reference to Fig. 5. At this location, stress convection is minor, and the high level of the pressure-velocity term has to be compensated by the observed increase in production. However, the rise in both processes, relative to the respective baseline levels, is not identical, and the difference is effective in just maintaining a shear stress level that is below that of the baseline case. As noted earlier, the unusual behaviour just described is linked to the peculiar response of the boundary layer to the sudden imposition of the actuation, and to the structure of the Stokes layer further downstream when $T^{+}$is far above the optimum value. This is discussed further below by reference to phase-averaged data.

Before this is done, however, a final set of time-averaged results is given in Figs. 7 and 8. These figure show, for $T^{+}=100, \mathrm{PDF}$ profiles for the streamwise and wall-normal fluctuations and joint $u^{\prime}-v^{\prime} \mathrm{PDF}$ contours at the streamwise positions ' 1 ' and ' 3 ', all again at the location $y^{+}=10$. In the latter set, the dashed lines pertain to the undisturbed baseline flow. Fig. 7 shows that there is an especially marked reduction in the breadth of the PDF of the streamwise fluctuations. The implication of the behaviour in the range $u^{\prime}<0$ and the shift of the maximum to the right-hand side is that this is due, preferentially, to a strong decline in the intensity of the low-speed streaks associated with ejections, coupled with an relative increase in the number of ejections. The PDF of the wall-normal fluctuations show a progressive decline in the intensity of both ejections and sweeps, consistent with reduced near-wall mixing. The symmetry of this PDF reflects the continuity constraint, but clearly, the effect of the reduced ejections is especially influential in respect of the streak intensity. This behaviour is essentially consistent with that observed in channel flow (Touber and Leschziner ${ }^{2}$ ). The joint-PDF contours reinforce the statements just made. As the flow progresses from position ' 1 ' to ' 3 ', there is, again, an especially strong reduction in the low-speed streak intensity. Events in the Q2 $\left(u^{\prime}<0, v^{\prime}>0\right)$ and Q4 $\left(u^{\prime}>0, v^{\prime}<0\right)$ quadrants are substantially reduced, consistent with a reduced shearstress levels - again driven primarily by the reduction in negative $u^{\prime}$ fluctuations associated with positive $v^{\prime}$ fluctuations. There is, furthermore, a noticeable anti-clockwise tilt in the contours, especially at location ' 1 ', suggesting a trend towards a reduced correlation between $u^{\prime}$ and $v^{\prime}$ fluctuations, and thus a reduction in shear stress over and above that implied by the narrowing of the PDFs in Fig. 7.

\section{B. Phase-averaged properties}

Attention is now turned to unsteady properties - that is, the response of phase-averaged quantities to the unsteady Stokes strain. A first aspect of the actuation that is important to highlight is the behaviour of the Stokes layer following the start of actuation. To this end, Stokes-flow profiles and the associated streamwise variations in the Stokes strain at $y^{+}=10$ are given in Fig. 9 for $T^{+}=100$. As seen from the profiles at location ' 1 ', only about $0.2 \delta_{0}$ downstream of the initiation of actuation, the Stokes layer has already reached $y^{+} \approx 10$, compared to a thickness $y^{+} \approx 15-20$ which the layer covers at the equilibrium (low-drag) state, downstream of station ' 6 '. It is well-understood, from channel-flow studies, that a high drag-reduction margin is contingent upon the Stokes layer remaining confined to the viscous sublayer $y^{+} \leq 15$ - which is the case at the optimum value $T^{+} \approx 100$ in channel flow. As is seen in the lower plot of Fig. 9, the Stokes strain at $y^{+}=10$ hardly changes in the streamwise 
direction beyond the location '2'. A notable exception is the dip in strain around location ' 4 ', and this reduction is associated with the upward penetration, locally, of the Stokes layer into the region beyond $y^{+} \approx 15$. The rapid establishment of the Stokes strain and its interaction with the relatively high level of turbulence activity just following the start of forcing is expected to cause strong phase-dependent fluctuations in the turbulence properties in the initial stages of the drag-reduction process. Importantly, it is observed in Fig. 9 that the Stokes layer at position ' 4 ' penetrates well beyond the viscous sublayer into the buffer layer above it, a condition that causes elevated spanwise-strain-induced production (of $w$ fluctuations), expected to result in pronounced periodic peaks in turbulence and drag at this location. As the flow progresses further downstream, the time-mean turbulence activity in the near-wall layer declines, the drag decreases and the Stokes layer eventually contracts towards $y^{+} \approx 15$. The expectation that the turbulence state around position ' 4 ' would show strong oscillatory characteristics associated with periodic peaks is confirmed in Fig. 10: both the phase-averaged skin-friction and turbulence fluctuations display moderately large amplitudes around position ' 4 '.

Fig. 10 shows, for $T^{+}=100$, that the phase-averaged $C_{f}$ features a distinct wavy motion between positions ' 1 ' and ' 5 '. This may not be obvious from the figure, because only two phase-averaged variations per property have been included for clarity. However, animations show, unambiguously, a wave-like propagation, with the wave length being around $0.7 \delta_{0}$. This will also emerge from a phase-space plot of $C_{f}$ (Fig. 14). This is, of course, a feature entirely absent in channel flow, except for a superficially similar behaviour following an impulsive start of an oscillatory actuation in a fully-developed baseline channel flow. The wave propagation speed can be readily extracted from Fig. 14 as being 10-12 $u_{\tau}$, or $U$, at $y^{+}=10-12$. With the above wave speed, it is simple to show that the time between two successive oscillation maxima is $t^{+} \approx 100$ - that is, the actuation period. Hence, while the generation of the oscillations is dictated by the actuation time scale, the propagation of the waves is not.

As shown in Fig. 10, similar and corresponding wavy behaviour is also observed in respect of the turbulence quantities and the streamwise shear strain at at $y^{+}=10$. Again, at location ' 4 ', the large amplitude in $C_{f}$ is well correlated with corresponding amplitudes of the stresses, with high/low values of $C_{f}$ associated with high/low values of the stresses. At this same location, the shear-strain fluctuations at $y^{+}=10$, while relatively modest, show the opposite correlation - i.e. reducing as the shear-stress increases, thus implying a flattening of the profile as turbulent mixing rises. It may be concluded, therefore, that large fluctuations generated at the onset of the actuation propagate by convection downstream and are subjected to oscillatory alterations by the Stokes strain, with the time-average turbulence and drag declining progressively. Around location ' 5 ', about $2.5 \delta_{0}$ beyond the start of the actuation, the wave-like oscillations die away, and $C_{f}$ experiences modest phaseinduced oscillations that are essentially uniform (homogeneous) in the streamwise direction.

The lowest plot in Fig. 10 shows the response of the phase-averaged wall-normal velocity and the associated stochastic stress component to the onset of the actuation. The response of the former is characterised, again, by a wavy motion that is well correlated with the shear strain fluctuations at the same location. While these oscillation are substantial, relative to the time-averaged level, the corresponding rms value is of order $1-2 \%$, and this component of the unsteady motion does not, therefore, contribute greatly to the total turbulent stress $\overline{v^{2}}$.

Distributions of the major contributors to the shear-stress budget at $T^{+}=100$ and $y^{+}=10$ are given in Fig. 11. The time-averaged distributions are those also contained in Fig. 5. Three noteworthy features in this figure are: (i) the fact that $P_{u v}$ reduces in line with the reduction in $\overline{v^{2}}$, notwithstanding the (modest) increase in the strain (see Fig. 10); (ii) the large peak in pressure-velocity interaction, $\phi_{u v}$, highlighted in Fig. 5 and discussed earlier, associated with large oscillations in this term immediately after the start of the actuation; and (iii) the modest oscillations in $P_{u v}$, relative to $\phi_{u v}$, corresponding to the equally modest oscillation in $\overline{v^{2}}$.

Phase-averaged variations for $T^{+}=200$, corresponding to those in Fig. 10 for $T^{+}=100$, 
are shown in Figs. 12, and 13, the latter together with four sets of Stokes-motion profiles at four streamwise locations. Although the details of these distributions differ from those at $T+=100$, the wavy-like motions downstream of the actuation are similar. One important point of difference relates to the region downstream of location ' 4 '. As for $T^{+}=100$, the shear-strain fluctuations are minor, and do not explain the rise of the shear-stress production above the baseline level beyond location ' 4 '. Moreover, as for $T^{+}=100$, fluctuations in the phase-averaged wall-normal velocity, while not fully converged, do not appear to contribute significantly to the total wall-normal stress; again, this stress is dominated by the stochastic component. However, in contrast to $T^{+}=100$, this component is seen to rise above the baseline level. This causes the shear-stress production to rise above that of the baseline value, leading to the gradual increase in the shear stress. As is evident from the Stokesstrain profiles, lower plots in Fig. 13, the above behaviour is associated with penetration of the Stokes strain well beyond the viscous sublayer at location ' 6 ' (and beyond). This causes the significant (nearly streamwise homogeneous) fluctuations in $C_{f}$ and the turbulent stresses beyond location ' 6 '.

The channel-flow study by Touber and Leschziner ${ }^{2}$, suggests that the near-wall streaks, the streamwise stress and the production of the latter are amplified during high Stokesstrain phase portions, around $y^{+}=10-15$, while the streaks are attenuated when the Stokes strain is relatively low and changes rapidly (i.e. the total strain vector changes its direction rapidly). The lag between maxima/minima in strain and maxima/minima in $C_{f}$ are observed to be around 60 degrees, reflecting the time scale associated with the response of the streak-decline/amplification process to the Stokes strain. Moreover, the streaks are indistinct (lacking clear orientation and structure) following phases of rapid rate of change in the Stokes strain, while the streaks reform, amplify and assume the orientation of the shear-strain angle during periods of high-strain and low-rate-of-change in the strain angle. Linear analysis by Blesbois et al. ${ }^{16}$ support the above observations, including the lag that, in channel flow, is associated with an amplification time scale of order $t^{+}=50$.

The applicability of the above characteristics to the present boundary-layer flows can only be examined by considering the minor fluctuations in the low-drag state following the transition region. This is done in the lower plot of Fig. 14, which shows phase- $x-$ space colour-contour maps of $C_{f}$ superimposed by line contours of the Stokes strain, $\partial W / \partial y$, all at $y^{+}=10$. While phase-dependent oscillations are weak in the low-drag state, beyond $x=8 \delta_{0}$, it is observed that there is, here too, a fairly close relationship between the Stokes strain and the oscillations in the examined flow properties. Thus, a rapid phase-wise (absolute) decline in the Stokes strain is associated with low $C_{f}$ values, while an increase towards a (positive or negative) maximum in strain is followed by a high $C_{f}$ value. The upper plot in Fig. 14 is a map of fluctuations in skin friction, $C_{f}^{(\phi)}-C_{f}^{\text {mean }}$, with line contours identifying the largest fluctuations in the region following the onset of the actuation. This plot has been referred to earlier in support of the argument that skin-friction oscillations propagate as waves having a wavelength of $0.7 \delta_{0}$ and a wave speed of $10-12 u_{\tau}$. The former can be inferred from the separation of the peaks at 7 and $7.7 \delta_{0}$, while the latter can be extracted from the slope of the contours.

Finally, Fig. 15 compares two snapshots of the streaky near-wall structure, expressed in terms of contours of streamwise fluctuations at $y^{+}=10$. One snapshot has been taken from the baseline flow and the other from the actuated flow, at $T^{+}=100$ at a phase in which the streaks are in the process of reforming at one of two distinct (left-right) orientations that Touber and Leschziner ${ }^{2}$ show to be closely associated with the extreme directions of the shear-strain vector around $y^{+} \approx 10$. The snapshot of the actuated flow shows clearly that, beyond the region immediately following the onset of actuation, the streak are oriented in the direction of the local strain vector, and animations confirm the near left-right switching of directions during the actuation cycle. Also evident are large regions of weak streaks, consistent with the low-drag state in which streamwise fluctuations are greatly reduced relative to the baseline. 


\section{CONCLUSIONS}

Although a channel flow and a boundary share some major characteristics in the way both respond to spanwise wall oscillations, there are also some significant differences between the two, and this limits the validity of using channel flow as a representative of boundary layers. This limitation is important to highlight, as the large majority of DNS studies in this area have focused on channel flow, exploiting the benefits of streamwise homogeneity to reduce costs. Above all, channel-flow simulations do not allow the transition towards the lowdrag state and back from that state toward the unactuated flow to be characterized when actuation is limited to a streamwise stretch of the boundary layer.

With attention first focused on the low-drag state, the present study strongly suggests that the maximum drag-reduction margin is lower, by as much as $5-7 \%$, in a boundary layer than in the equivalent channel flow. Moreover, the optimum actuation period, at which the maximum drag reduction is achieved, is significantly lower, perhaps by as much as $40 \%-$ around $T^{+}=70$, relative to $100-120$ in channel flow. At $T^{+}=200$, the largest actuation period examined, the low-drag skin friction is still marginally below the baseline value, but the wall-normal stress and the shear-stress production exceed the corresponding baseline levels, due to the adverse effects of the Stokes layer penetrating the turbulent region above the viscous sublayer. In contrast, in channel flow, this unfavourable behaviour does not occur until $T^{+}$is raised to around 400. While the underlying reasons for these differences are not elucidated in this study, is it conceivable that the very different outer structures in a channel and a boundary layer play a role in the differences in the response of the near-wall turbulence to the actuation. This is suggested by the non-negligible Reynoldsnumber dependence of the drag-reduction margin observed in channel flow, which is strongly suspected to be rooted in the effects of the footprints of large outer structures on the nearwall streaks ${ }^{2}$ and the modulation of the near-wall streaks they cause. Another difference observed between channel flow and the present boundary layer is that the fairly large phasedependent oscillations in skin friction around the low-drag state, observed in channel flow at non-optimum actuation periods, are considerably weaker in the boundary layer. Despite this weakness, it has been possible to confirm that the skin friction and the turbulent stresses in the viscous sublayer reach corresponding maxima following a phase, within any one actuation cycle, in which the Stokes strain at around $y^{+}=10$ rises towards its maximum, while the skin friction reaches a minimum following a phase in which the Stokes strain declines. In common with channel flow, the streaky structure in a boundary layer shows a close-to-bimodal orientation angle during the actuation cycle, a behaviour that is closely linked to the response of the streaks to the shear-strain angle at around $y^{+}=10$ around which the streaks are formed and are at their strongest.

The transition towards the low-drag state, following the start of the actuation, was found to occupy around 5 boundary-layer thicknesses, corresponding to around 3 actuation periods, when evaluated with the convection velocity at $y^{+}=10$. The recovery from the low-drag state is similarly fast. These observations concur, broadly, with those of Skote ${ }^{10}$. They are important from a practical perspective, in so far as they demonstrate that control effectiveness is weak and short-lived outside the region in which the actuation is effected.

An entirely new feature brought out by the phase-averaged analysis included in the paper is the presence of substantial oscillations in the skin friction and near-wall turbulence properties that are provoked by the onset of the actuation, and which propagate convectively in a wave-like manner with a velocity $U^{+} \approx 10$. These waves, which are entirely uncorrelated with the spanwise strain, decay over a distance of around 4-5 boundary layer thicknesses as the flow approaches the low-drag state. The oscillations in the turbulent stresses are correlated with corresponding oscillations of their respective phase-averaged production rates. A mechanism that appears to be especially sensitive to the sudden onset of the actuation is the pressure-velocity interaction in the shear stress budget. This shows large oscillations, immediately after the start of the actuation, reflected by a strong spike in its time-averaged value. The imbalance between this term and the production term, which oscillates more weakly, is compensated by significant fluctuations in the convection of the shear stress. 


\section{Acknowledgements}

The provision of super-computer resources on HeCTOR, via a grant from EPSRC UK, is gratefully acknowledged.

${ }^{1}$ M. Quadrio, P. Ricco, and C. Viotti, "Streamwise-travelling waves of spanwise wall velocity for turbulent drag reduction," Journal of Fluid Mechanics 627, 161-178 (2009)

${ }^{2}$ E. Touber and M. Leschziner, "Near-wall streak modification by spanwise oscillatory wall motion and drag-reduction mechanisms," Journal of Fluid Mechanics 693, 150 (2012)

${ }^{3}$ F. Laadhari, L. Skandaji, and R. Morel, "Turbulence reduction in a boundary layer by a local spanwise oscillating surface," Physics of Fluids 6, 3218-3220 (1994)

${ }^{4}$ K. Choi, J. DeBisschop, and B. Clayton, "Turbulent boundary-layer control by means of spanwise-wall oscillation," AIAA journal 36 (1998)

${ }^{5} \mathrm{~K}$. Choi, "Near-wall structure of turbulent boundary layer with spanwise-wall oscillation," Physics of Fluids 14, 2530 (2002)

${ }^{6}$ G. Di Cicca, G. Iuso, P. Spazzini, and M. Onorato, "Particle image velocimetry investigation of a turbulent boundary layer manipulated by spanwise wall oscillations," Journal of Fluid Mechanics 467, 41-56 (2002)

${ }^{7} \mathrm{P}$. Ricco, "Modification of near-wall turbulence due to spanwise wall oscillations," Journal of Turbulence (2004)

${ }^{8}$ K. Choi and M. Graham, "Drag reduction of turbulent pipe flows by circular-wall oscillation," Physics of Fluids 10, 7 (1998)

${ }^{9}$ M. Skote, "Turbulent boundary layer flow subject to streamwise oscillation of spanwise wall-velocity," Physics of Fluids 23, 081703 (2011)

${ }^{10} \mathrm{M}$. Skote, "Temporal and spatial transients in turbulent boundary layer flow over an oscillating wall," International Journal of Heat and Fluid Flow 38, 1-12 (2012)

${ }^{11}$ N. Kasagi, Y. Suzuki, and K. Fukagata, "Microelectromechanical systems-based feedback control of turbulence for skin friction reduction," Annual Review of Fluid Mechanics 41, 231-251 (2009)

${ }^{12} \mathrm{~B}$. Frohnapfel, Y. Hasegawa, and M. Quadrio, "Money versus time: evaluation of flow control in terms of energy consumption and convenience," Journal of Fluid Mechanics 700, 406-418 (2012)

${ }^{13}$ M. Quadrio and P. Ricco, "Critical assessment of turbulent drag reduction through spanwise wall oscillations," Journal of Fluid Mechanics 521, 251-271 (2004)

${ }^{14}$ G. Karniadakis and K. Choi, "Mechanisms on transverse motions in turbulent wall flows," Annual Review of Fluid Mechanics 35, 45-62 (2003)

${ }^{15}$ J. Choi, C. Xu, and H. Sung, "Drag reduction by spanwise wall oscillation in wall-bounded turbulent flows," AIAA journal 40, 842-850 (2002)

${ }^{16}$ O. Blesbois, S. I. Chernyshenko, E. Touber, and M. Leschziner, "Pattern prediction by linear analysis of turbulent flow with drag reduction by wall oscillation," Journal of Fluid Mechanics 724, 607-641 (2013)

${ }^{17} \mathrm{M}$. Quadrio and P. Ricco, "Initial response of a turbulent channel flow to spanwise oscillation of the walls," Journal of Turbulence (2003)

${ }^{18} \mathrm{C} . \mathrm{Xu}$ and $\mathrm{W}$. Huang, "Transient response of reynolds stress transport to spanwise wall oscillation in a turbulent channel flow," Physics of Fluids 17, 018101 (2005)

${ }^{19}$ P. Schlatter, R. Örlü, Q. Li, G. Brethouwer, J. Fransson, A. Johansson, P. Alfredsson, and D. Henningson, "Turbulent boundary layers up to $R e_{\tau}=2500$ studied through simulation and experiment," Physics of Fluids 21, 051702 (2009)

${ }^{20} \mathrm{~S}$. Lardeau and M. Leschziner, "The interaction of round synthetic jets with a turbulent boundary layer separating from a rounded ramp," Journal of Fluid Mechanics 683, 172 (2011)

${ }^{21}$ Y. Bentaleb, S. Lardeau, and M. Leschziner, "Large-eddy simulation of turbulent boundary layer separation from a rounded step," Journal of Turbulence (2012)

${ }^{22}$ J. Jiménez, S. Hoyas, M. P. Simens, and Y. Mizuno, "Turbulent boundary layers and channels at moderate reynolds numbers," Journal of Fluid Mechanics 657, 335-360 (2010)

${ }^{23} \mathrm{X}$. Wu and P. Moin, "Transitional and turbulent boundary layer with heat transfer," Physics of Fluids 22, $085105(2010)$ 

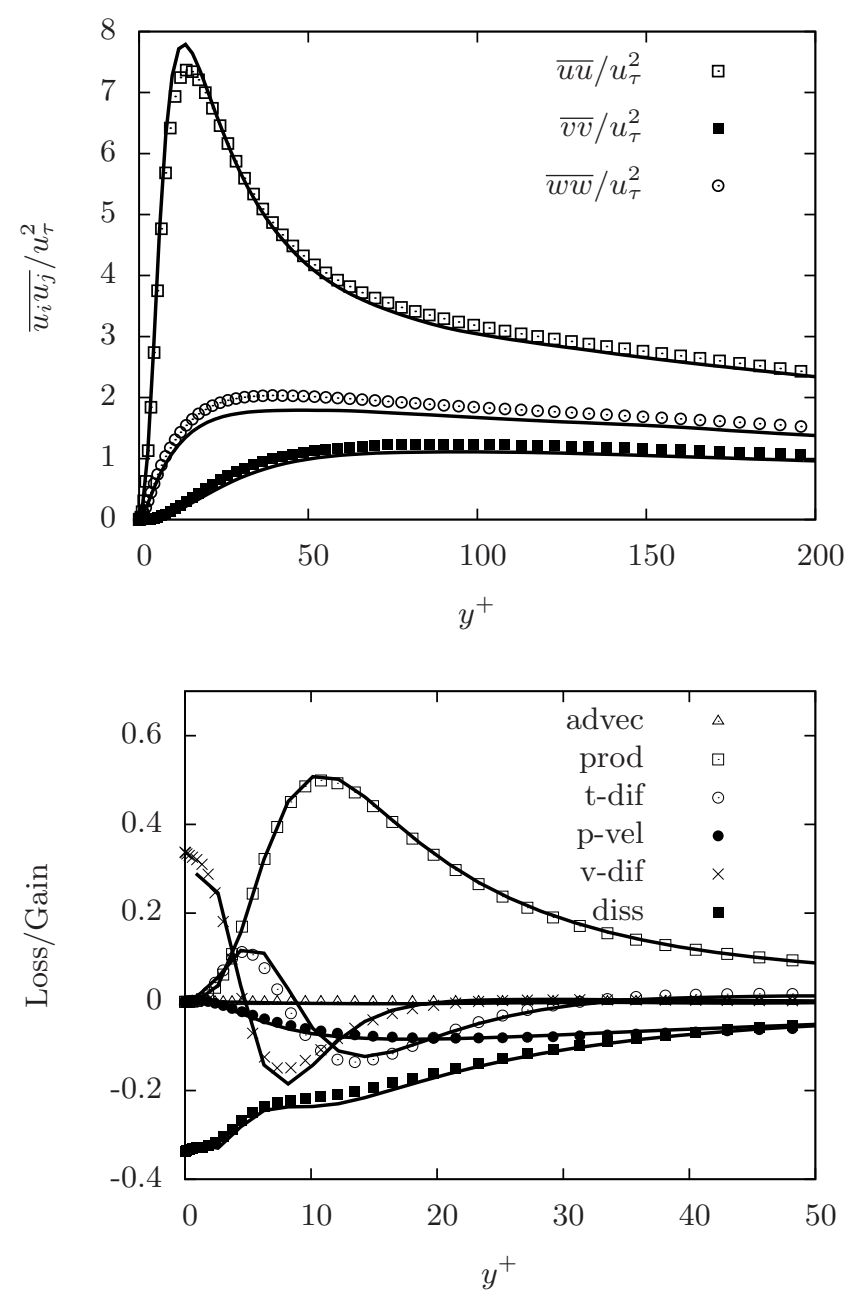

FIG. 1. Wall-scaled Reynolds stresses and budget of $\overline{u u}$. Solid lines: present computation at $R e_{\delta^{*}}=1230$; symbols: Schlatter et al. ${ }^{19}$ at $\operatorname{Re}_{\delta^{*}}=1410$. 

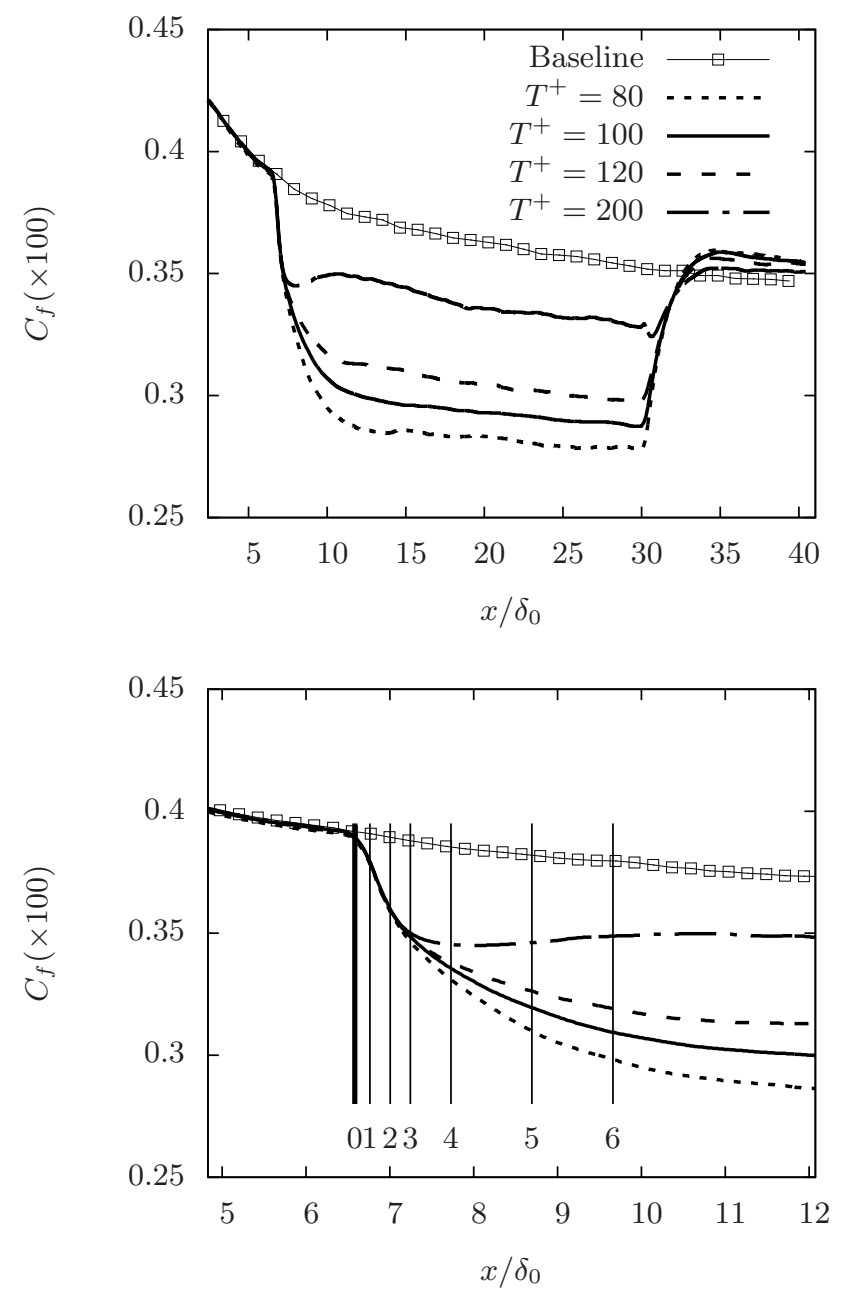

FIG. 2. Streamwise evolution of skin-friction coefficient $C_{f}$. Lower plot gives a magnified view of the upstream transition portion, with specific streamwise stations featuring in the discussion and results to follow. 

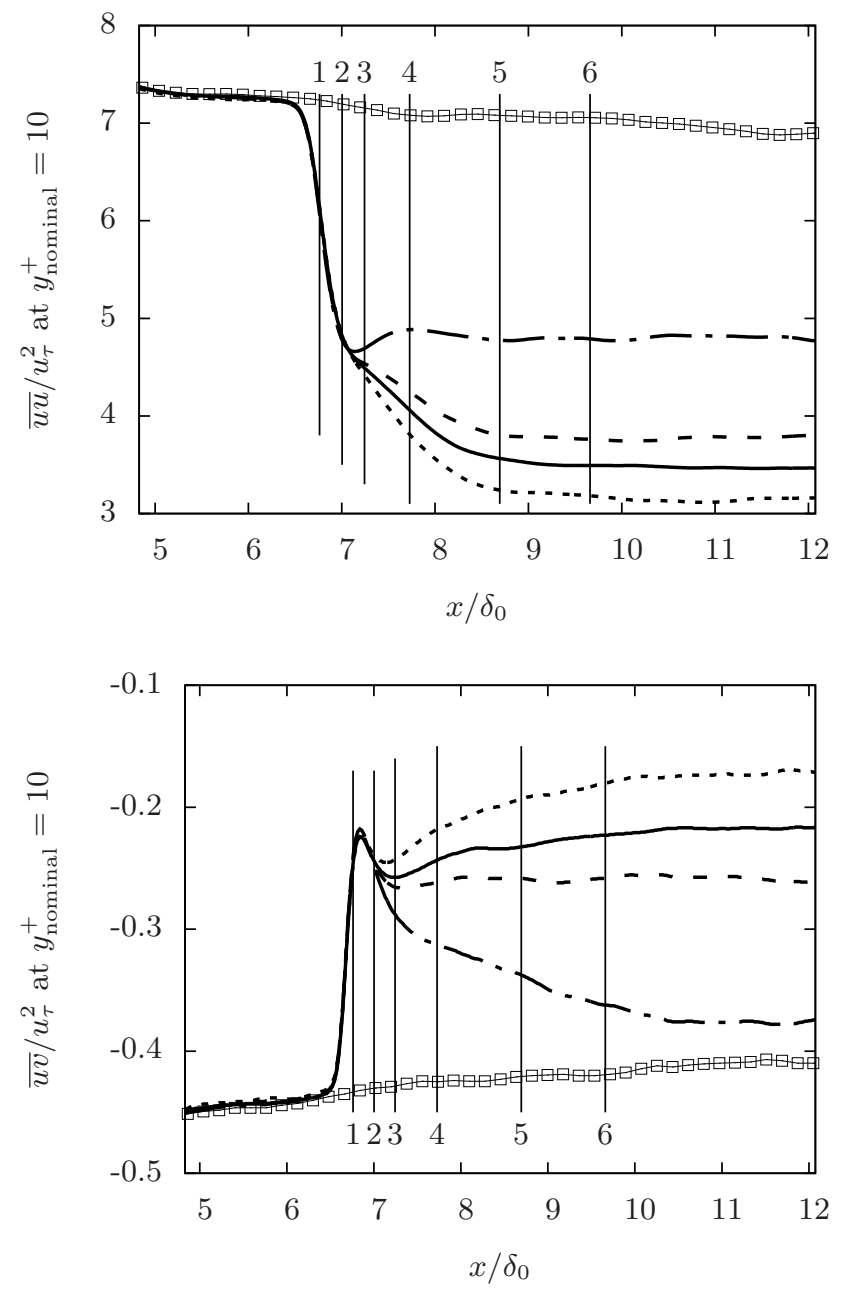

FIG. 3. Streamwise evolution of the streamwise-normal stress $\overline{u u}$ and shear-stress $\overline{u v}$ at $y^{+}=10$. The key to lines is given in Fig. 2 

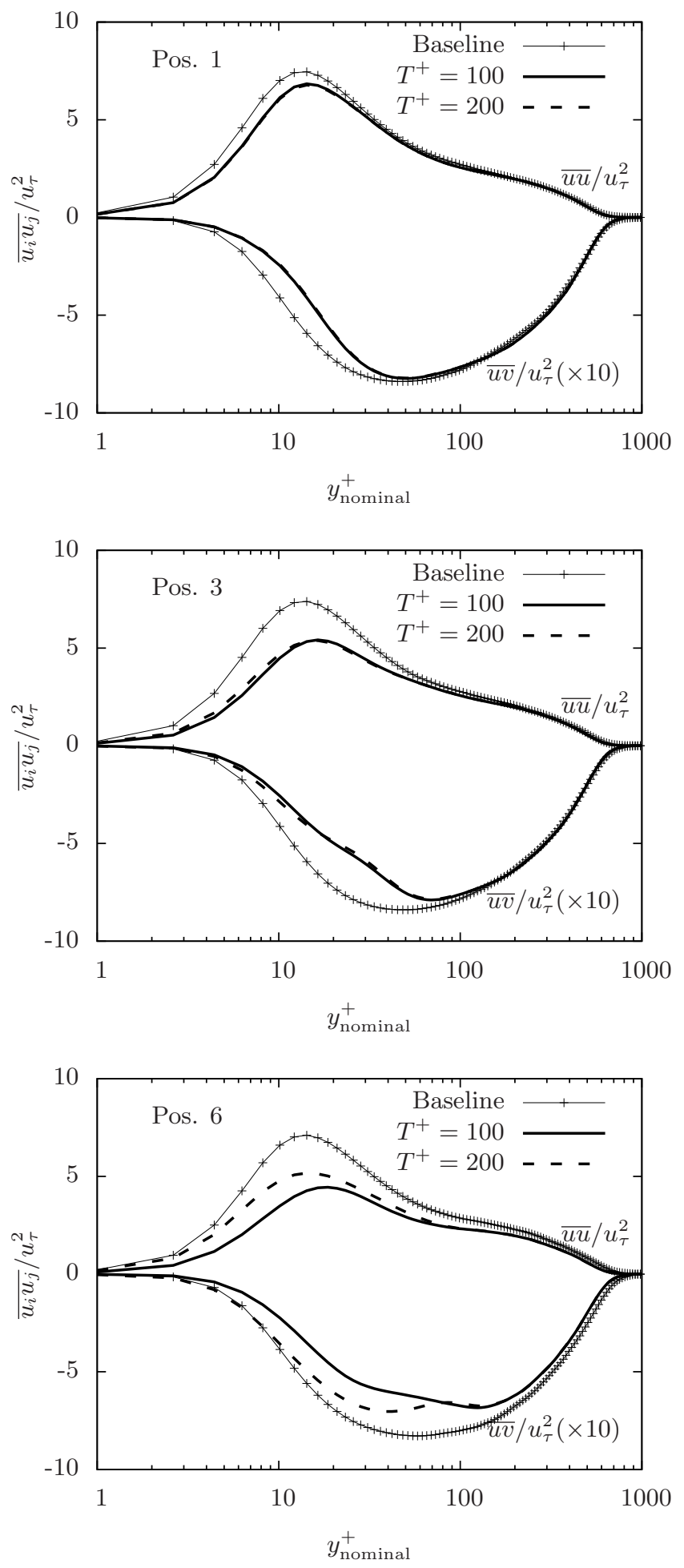

FIG. 4. Profiles of streamwise normal stress $\overline{u u} / u_{\tau}^{2}$ and shear stress $\overline{u v} / u_{\tau}^{2}$ for the positions ' 1 ', ' 3 ' and ' 6 ' (cf fig. 2). 

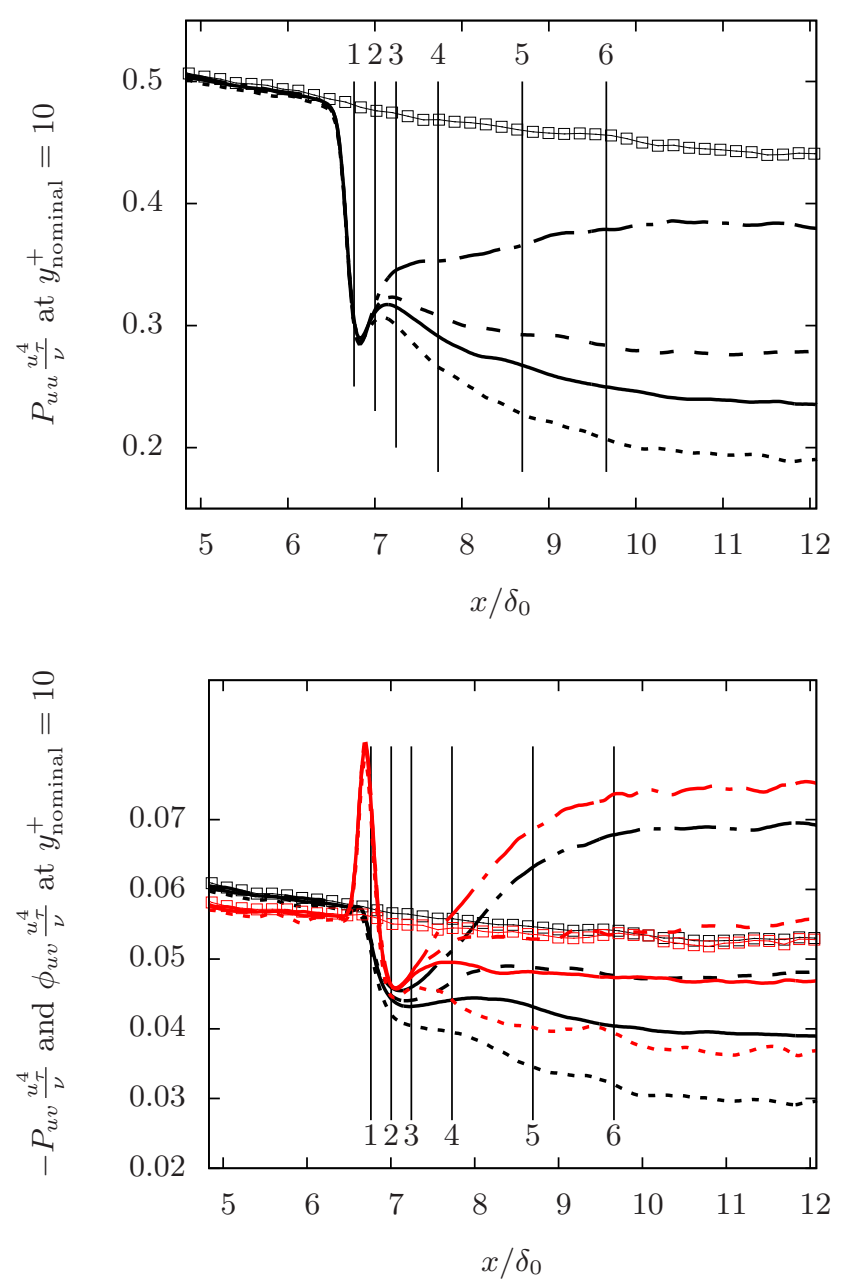

FIG. 5. Streamwise variation of the production terms of the stresses shown in Fig. 3. Variations of the the pressure-velocity-interaction term are included with red lines in the case of the shear stress. The key to lines in given in Fig. 2 

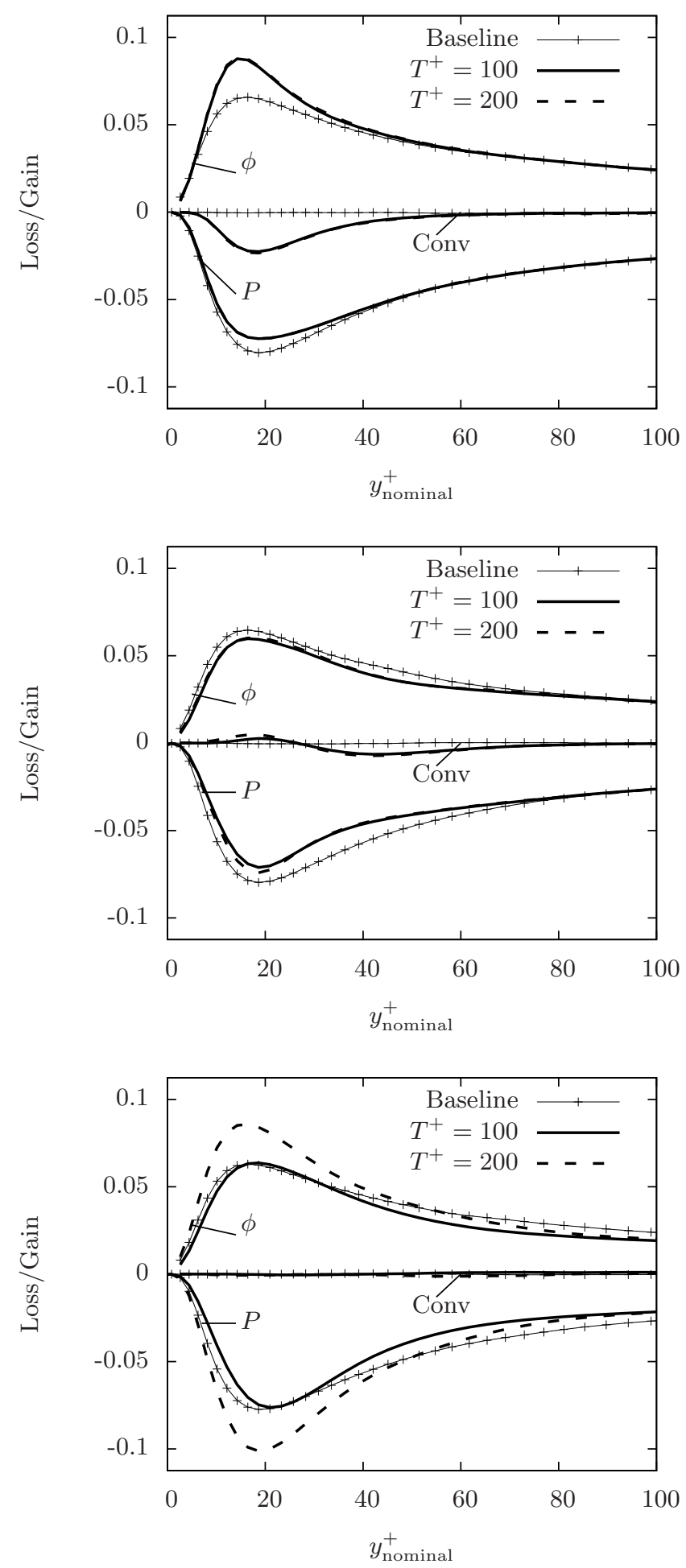

FIG. 6. Profiles of the leading terms in the balance of the shear stress $\overline{u v}$ for positions ' 1 ', ' 3 ' and ' 6 ' (cf. Fig. 2). Normalization is done in inner scaling, as in Fig. 5. 

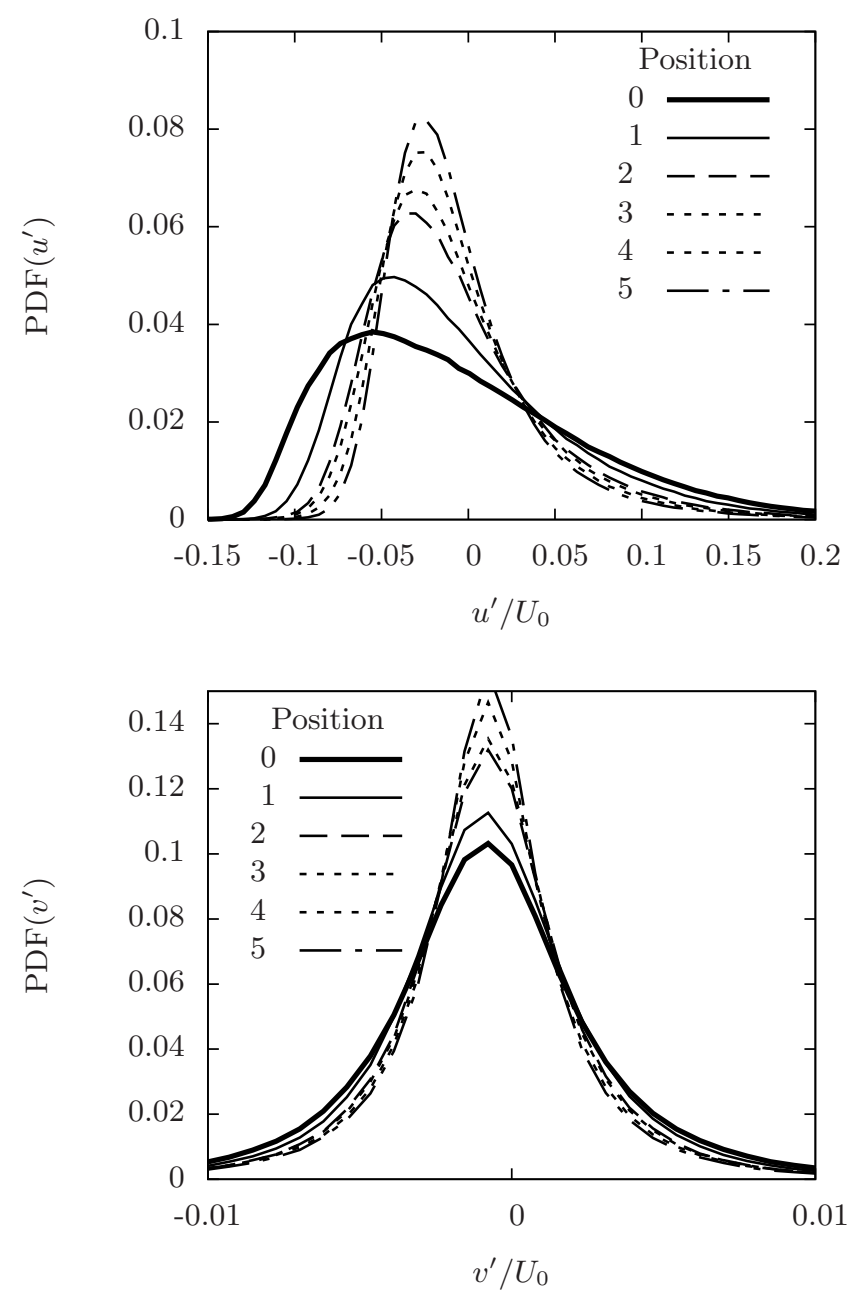

FIG. 7. PDFs for $u^{\prime}$ and $v^{\prime}$ at various streamwise locations for $T^{+}=100$. All PDFs are normalised to yield unit area 
Position 1

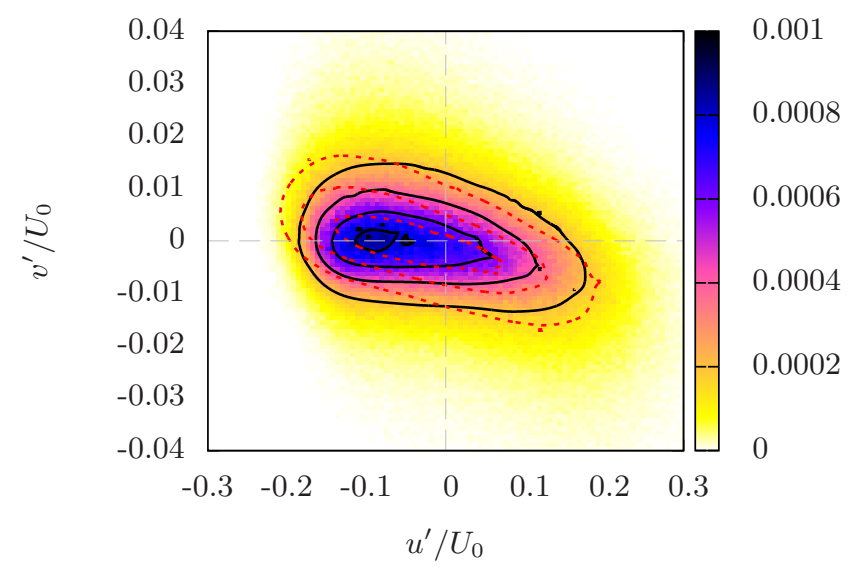

Position 3

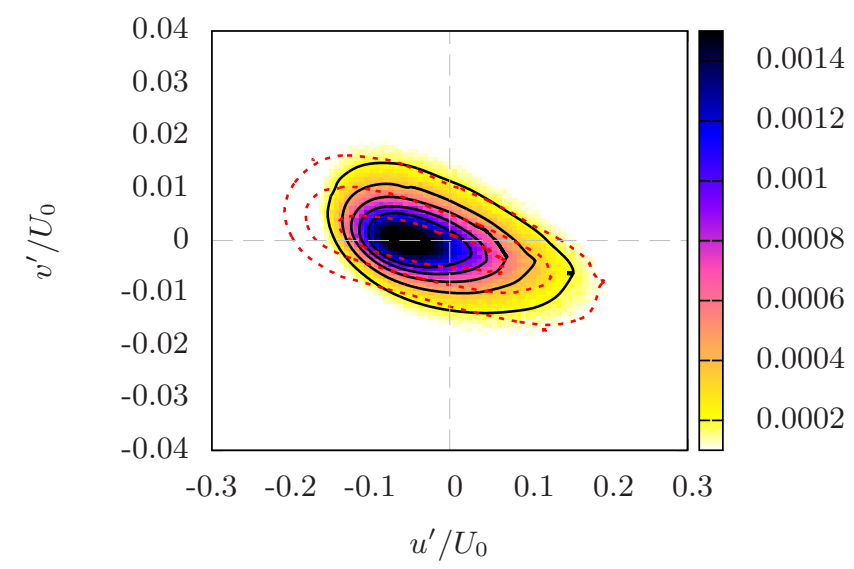

FIG. 8. Contours of joint PDF of $u$ and $v$ for $T^{+}=100$ at $y^{+}=10$ at two streamwise positions (see Fig. 2). Dashed contours relate to the unperturbed flow. 

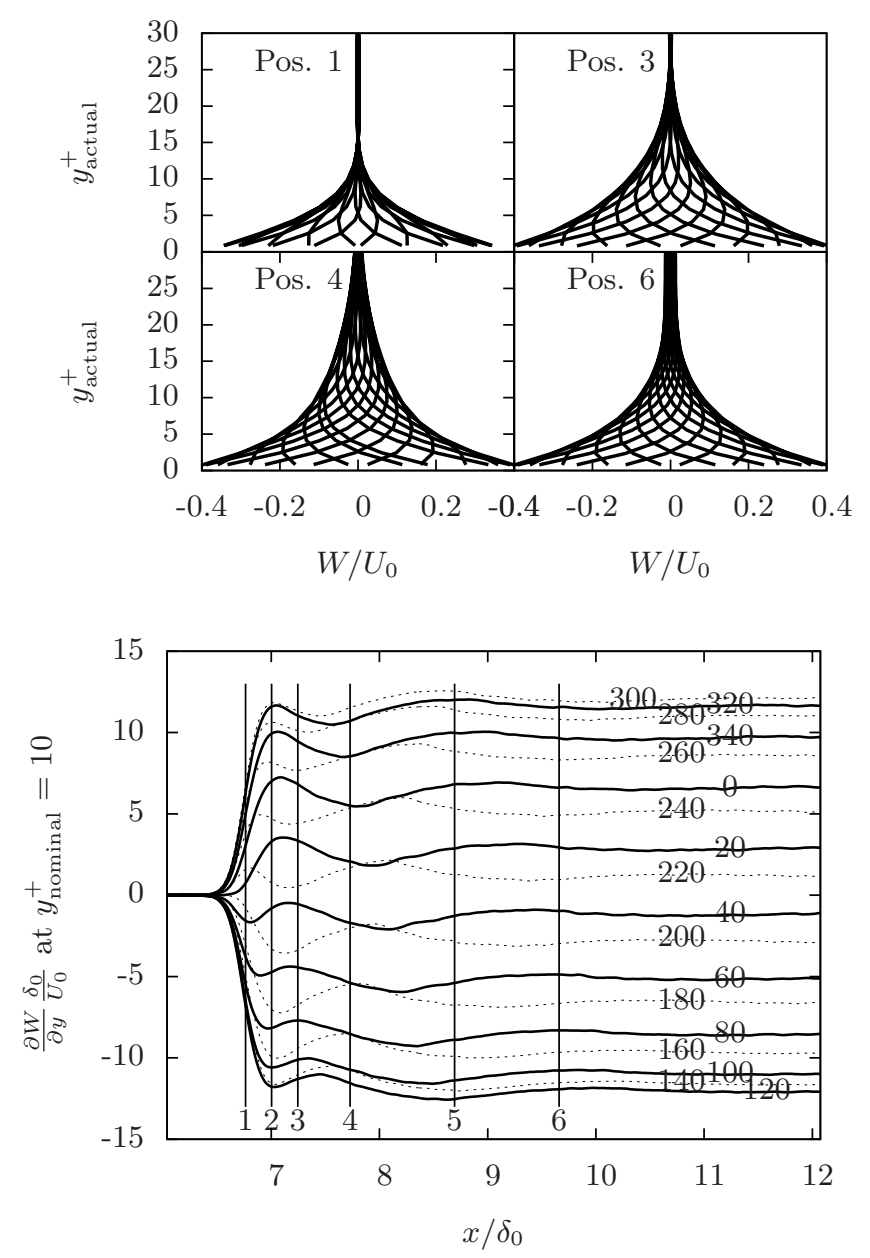

FIG. 9. Phase-averaged spanwise velocity at four streamwise locations (upper plot) and streamwise evolution of the spanwise strain at various phase values for $T^{+}=100$ (lower plot). 

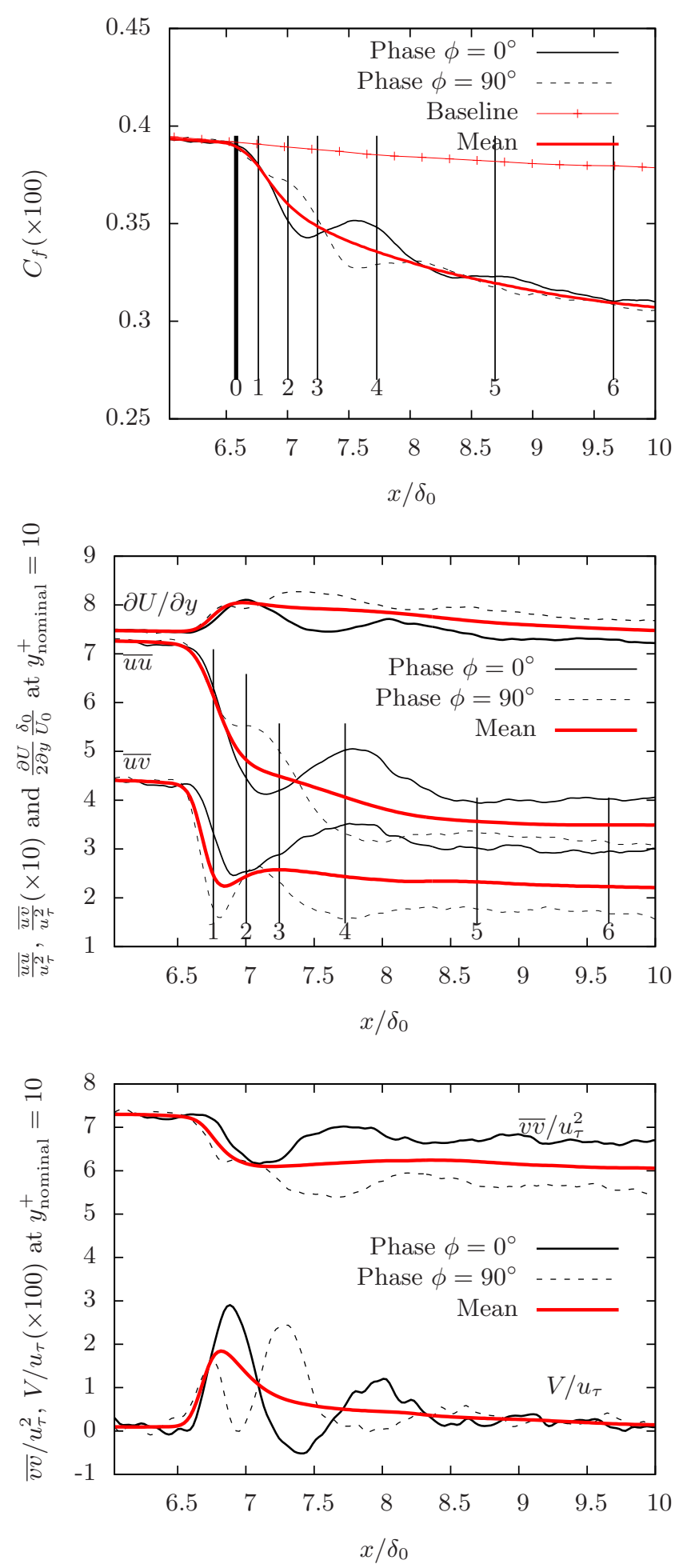

FIG. 10. Streamwise variations of phase-averaged and time-mean properties for $T^{+}=100$ and $y^{+}=10$. From top to bottom: skin-friction coefficient, streamwise shear strain, streamwise normal stress, shear stress, wall-normal stress and wall-normal velocity. 


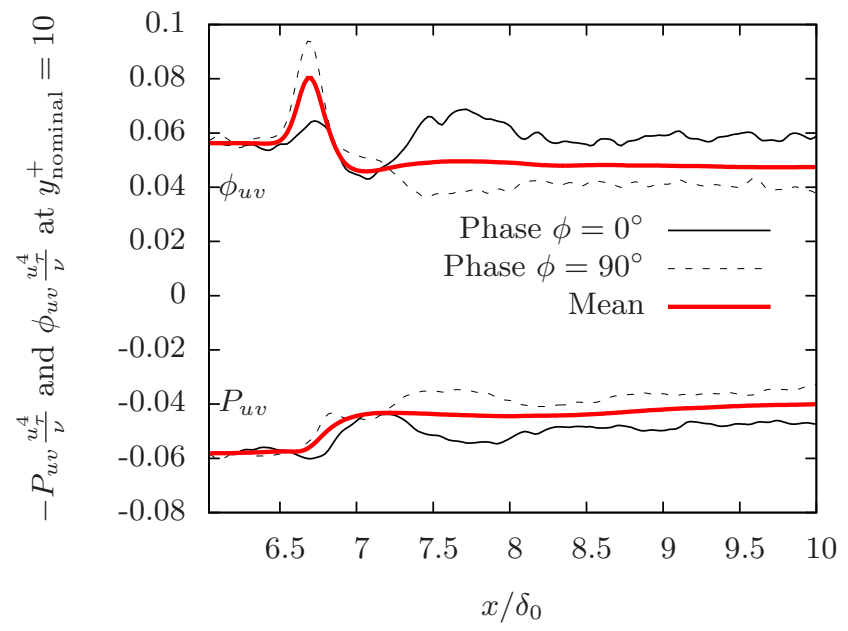

FIG. 11. Streamwise variations of principal phase-averaged and time-mean contributions to the shear-stress budget for $T^{+}=100$. 

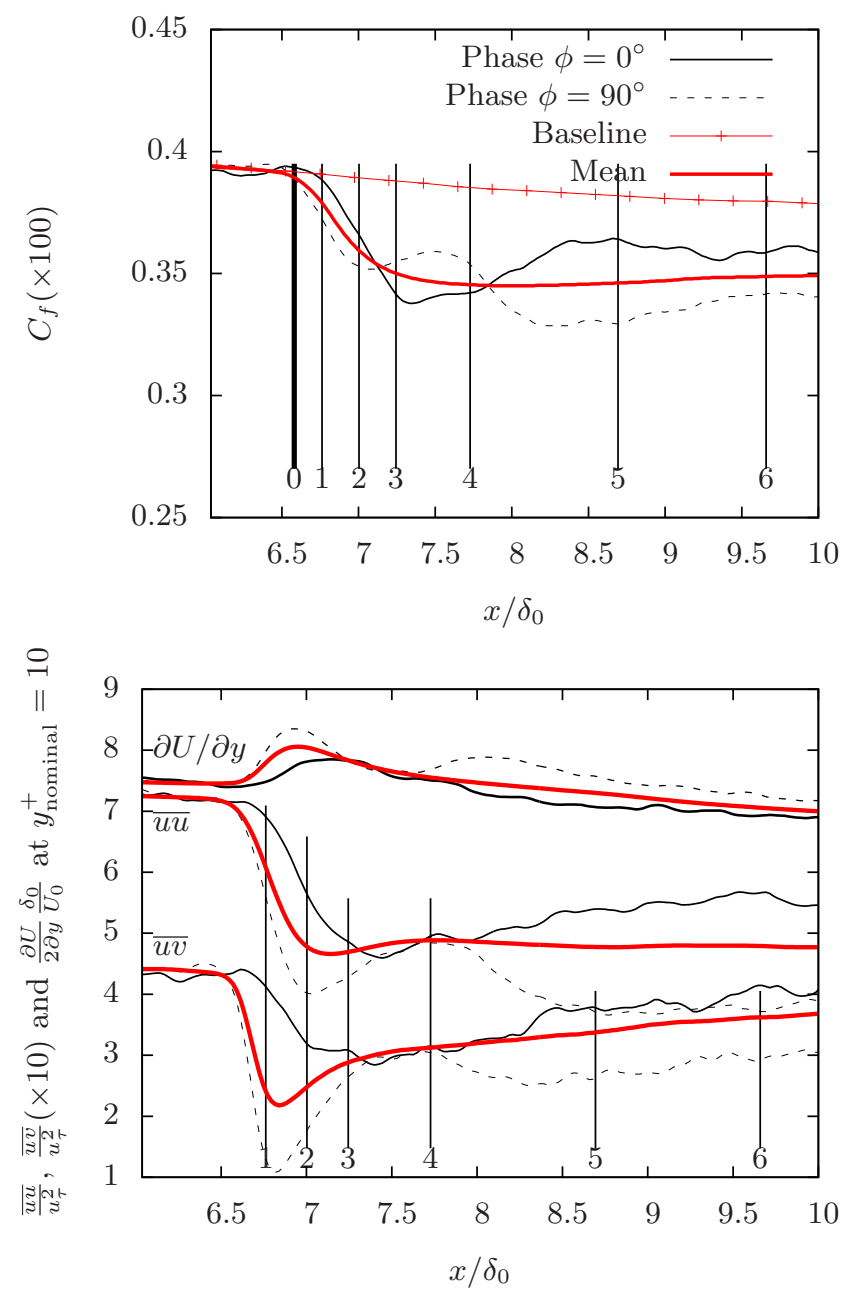

FIG. 12. Streamwise variations of phase-averaged and time-mean properties for $T^{+}=200$ and $y^{+}=10$. From top to bottom: skin-friction coefficient, streamwise shear strain, streamwise normal stress and shear-stress. 

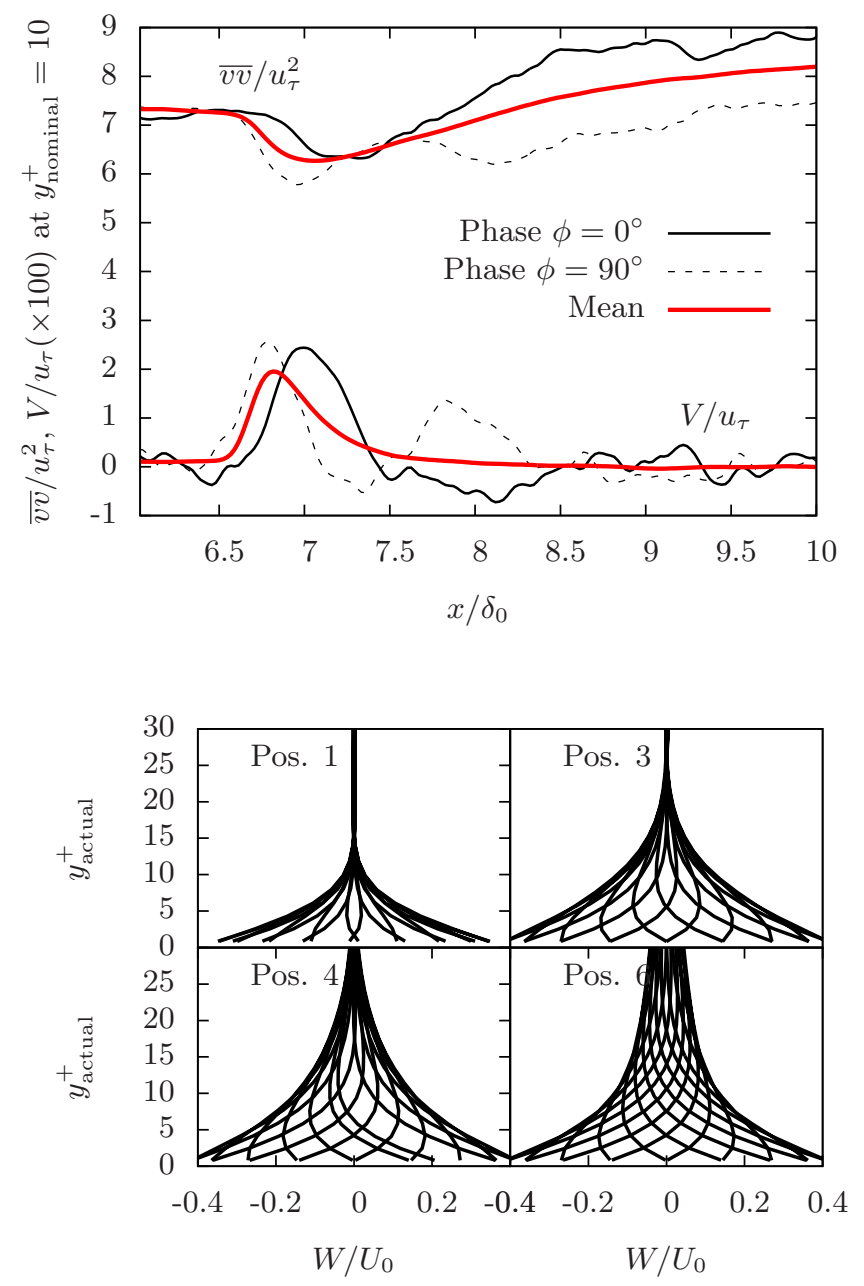

FIG. 13. Streamwise variations of phase-averaged and time-mean wall-normal stress and wallnormal velocity for $T^{+}=200$ (upper plot); and wall-normal profiles of spanwise velocity at four different streamwise locations for $T^{+}=200$ (lower plot). 

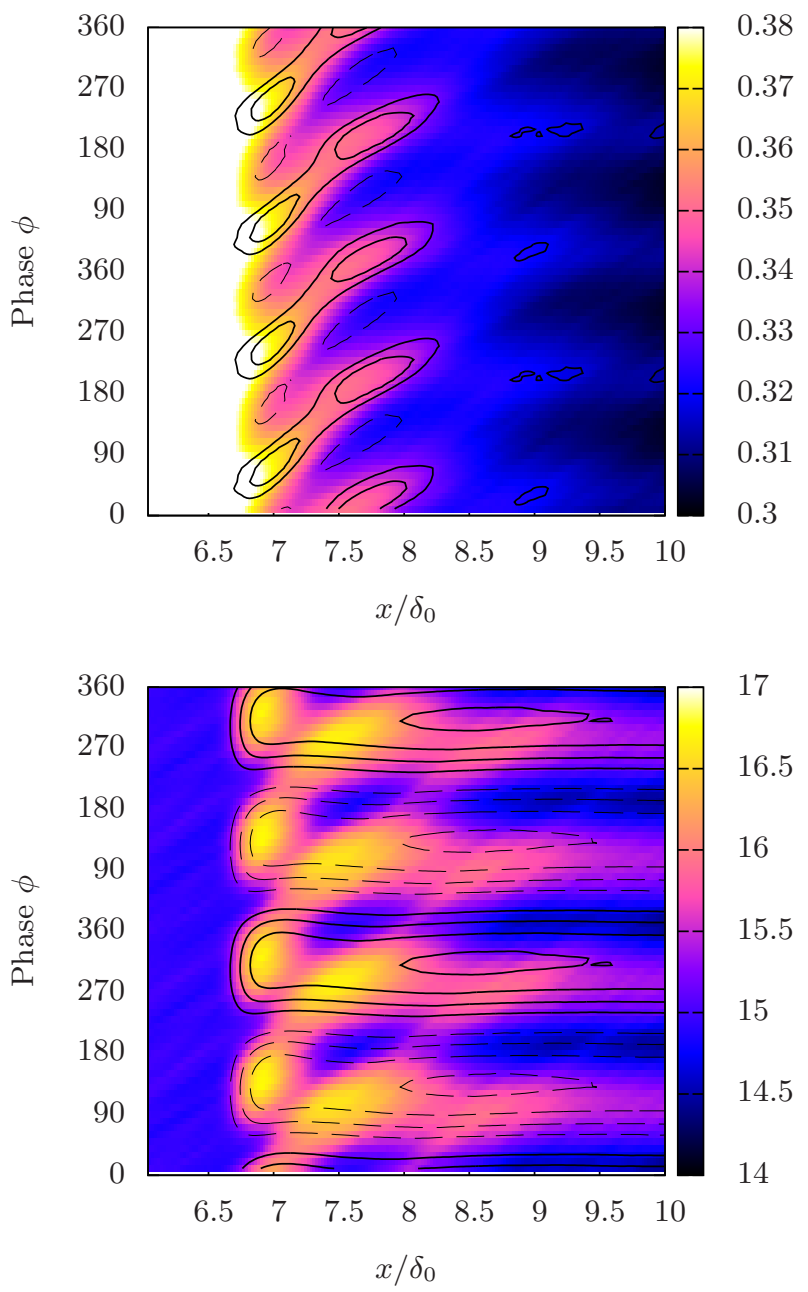

FIG. 14. Phase-averaged contours of skin-friction coefficient $C_{f}$. Upper plot: colour contours indicate skin-friction fluctuations $\left(C_{f}^{(\phi)}-C_{f}^{\text {mean }}\right)$; line contours represent specific levels associated with large fluctuations. Lower plot: colour contours indicate skin friction, $C_{f}$; line contours indicate iso-values of $\partial W / \partial y$ at $y^{+}=10$; positive values $\left(\partial W / \partial y \delta_{0} / U_{0}=3,6,9\right.$ and 15$)$ are shown as solid lines and corresponding negative values as dashed lines. 


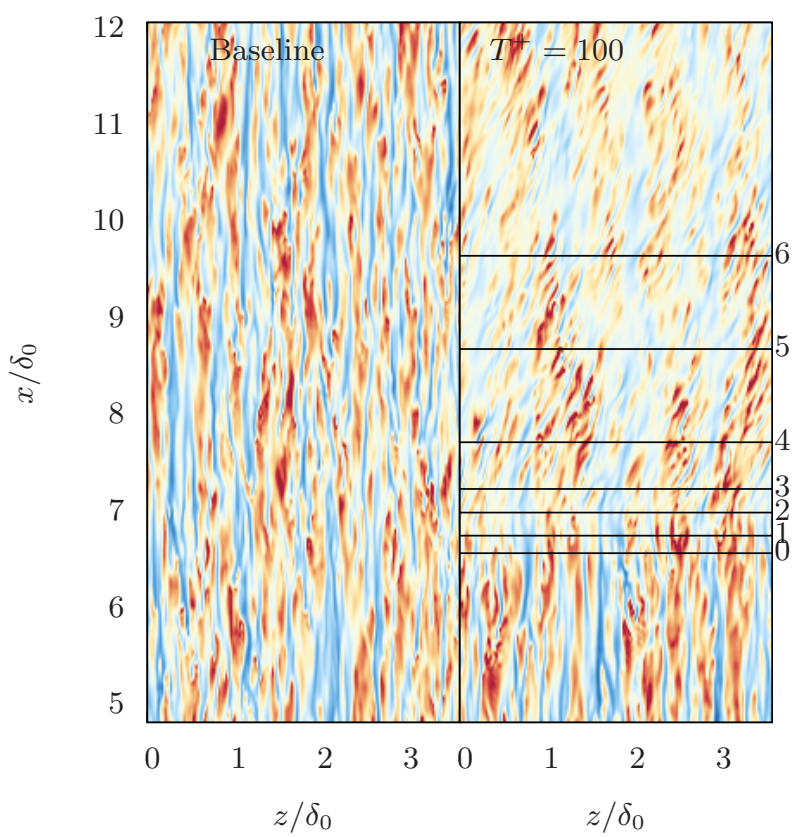

FIG. 15. Streamwise velocity fluctuation $u^{\prime}$ in a plane parallel to the wall, at $y_{\text {nominal }}^{+}=10$. Contours of $u^{\prime} / U_{0}$ vary from -0.1 to 0.1 . 